\title{
APLICAÇÃO DE MODELOS MATEMÁTICO-NUMÉRICOS PARA CÁLCULO DE PERFIS NOS LAMINADORES DE TIRAS A FRIO DA COMPANHIA SIDERÚRGICA NACIONAL*
}

\author{
Yukio Shigaki ${ }^{1}$ \\ Eduardo Amorim Motta ${ }^{2}$ \\ Willian Costa do Nascimento ${ }^{3}$
}

\section{Resumo}

Tendo como objetivo a melhoria contínua dos processos de laminação de produtos planos, foi elaborado um projeto de aplicação de modelos matemático-numéricos para a simulação dos laminadores de tiras a frio da Companhia Siderúrgica Nacional (CSN). A premissa principal é a de que esses modelos permitirão relacionar as diversas variáveis operacionais com o perfil emergente da tira e, baseado na variação da coroa entre a entrada e a saída, estimar a qualidade da sua planicidade. O presente trabalho apresenta os principais modelos aplicados, informações sobre os ensaios realizados para tomada dos dados industriais e alguns dos primeiros resultados com foco no perfil e planicidade. Resultados preliminares indicaram a modificação da coroa dos cilindros de trabalho do Laminador de Encruamento, levando à redução da quantidade de tiras com ondulado lateral. Também foram obtidos resultados satisfatórios na simulação das cadeiras do Laminador de Tiras a Frio no. 1, que é composto de cadeiras 6-high e 4-high.

Palavras-chave: Laminação de tiras a frio; Perfil; Simulação; Elementos finitos.

\section{APPLICATION OF MATHEMATICAL AND NUMERICAL MODELS FOR CALCULATION OF PROFILE FOR COLD ROLLING MILLS OF COMPANHIA SIDERÚRGICA NACIONAL}

\begin{abstract}
Having a continuous improvement objective of flat rolling processes, a project was developed to apply mathematical-numerical models for the simulation of the cold rolling mills at Companhia Siderúrgica Nacional (CSN). The main premise is that these models will allow to understand the behavior of strip crown when operational variables are changed and, based on the variation of the crown before and after rolling, to assess the quality of the flatness of the strip. This work presents the main models applied, the tests carried out to collect industrial data and some of the first results focused on the strip profile and its flatness. Preliminary results showed that a modification of the crown of the working rolls of the Temper Rolling Mill, led to a reduction of the number of strips with edge waves. Satisfactory results were also obtained in the simulation of the stands of the Tandem Cold Rolling Mill 1, which has 6-high and 4-high mills.
\end{abstract}

Keywords: Cold strip rolling; Strip profile, Simulation; Finite element method.

1 Engenheiro Naval, Doutor em Engenharia Metalúrgica pela UFMG, Professor universitário, Departamento de Engenharia Mecânica, CEFET-MG, Belo Horizonte, Minas Gerais, Brasil.

2 Engenheiro de Metalúrgico, Mestre em Engenharia de Materiais pela EEL / USP, Gerência Geral de Folhas Metálicas, Companhia Siderúrgica Nacional, Volta Redonda, Rio de Janeiro, Brasil.

3 Engenheiro de Metalúrgico, Mestre em Engenharia Metalúrgica pela UFF/EEIMVR, Gerência de Processos de Laminação, Companhia Siderúrgica Nacional, Volta Redonda, Rio de Janeiro, Brasil. 


\section{INTRODUÇÃO}

Diante da crescente exigência por parte dos consumidores, têm-se feito grandes investimentos no desenvolvimento de equipamentos mais eficientes na fabricação de produtos planos e não planos que tenham características geométricas e mecânicas com tolerâncias cada vez mais restritas. Diante disso, diversas empresas siderúrgicas têm investido na modernização de suas plantas de produção, visando maior produtividade e competitividade, preocupados em não perder mercado interno e em conquistar clientes externos.

Para uma tira ser considerada satisfatória, esta não deve possuir somente uma espessura uniforme ao longo de seu comprimento, mas também, tanto quanto possível, deve possuir um perfil transversal uniforme e deve estar isenta de ondulações. O perfil transversal tem sido chamado, por vezes, de "coroa" e, a planicidade pode ser chamada também de "forma".

Com a finalidade de atender aos mais exigentes padrões de qualidade, a Companhia Siderúrgica Nacional (CSN) tem empreendido um contínuo esforço por elevar o conhecimento tecnológico de seus processos de produção de tiras por meio do desenvolvimento de modelos matemáticos e computacionais.

A simulação dos processos de laminação de produtos planos permite relacionar as diversas variáveis operacionais e de produção com alguns parâmetros mecânicos e geométricos da tira laminada, tal como a coroa emergente em uma cadeira ou em um laminador tandem.

Em 2013 foi aprovado no Edital 94/2013 do Conselho Nacional de Desenvolvimento Científico e Tecnológico (CNPq), um projeto de pesquisa do Centro Federal de Educação Tecnológica de Minas Gerais (CEFET-MG) em parceria com a CSN, intitulado "Análise do defeito de planicidade em chapas laminadas e possíveis soluções no Laminador de Tiras a Frio no. 1 da Companhia Siderúrgica Nacional ", tendo como objetivo geral analisar esse tipo de defeito, compreender as fontes de seu surgimento e buscar possíveis soluções de modo a elevar a competitividade da indústria nacional frente aos produtos concorrentes externos, conforme preconiza o Plano Brasil Maior (PBM). Apesar do título citar somente o Laminador de Tiras a Frio no. 1 (LTF1), também foram incluídos os Laminadores de Encruamento no presente estudo.

Concretamente, visou-se:

- ajustar o modelo matemático MCl/Berger (desenvolvido pelo primeiro autor em seu Doutorado ${ }^{1}$ ) ao trem laminador de chapas a frio da CSN, incluindo controle de roll bending;

- ajustar um modelo dos laminadores 6-high e 4-high via método híbrido MEF (Método dos Elementos Finitos) 3D/Blocos; e,

- associar o controle de roll bending ao controle de planicidade de chapas.

Serão apresentados em seguida os modelos aplicados, informações sobre os ensaios realizados para tomada dos dados industriais e alguns dos primeiros resultados com foco no perfil e planicidade.

Referências mais atuais modelam o laminador e a tira completamente via elementos finitos $^{2}$, porém mostram-se pouco viáveis do ponto de vista prático quando se considera o excessivo tempo de processamento computacional para cada caso (cerca de 52 horas, segundo a mesma referência).

Os dois modelos deste trabalho simulam com maior velocidade por se tratarem de simuladores híbridos, acoplando modelos de deformação dos cilindros e modelos de cálculos de carga. Particularmente, o modelo MEF 3D/Blocos se mostra interessante 
para a situação em que se deseja laminar tiras cada vez mais finas e de maior resistência à deformação (os chamados aços - Advanced High Strength Steels AHSS) e na simulação de laminadores mais sofisticados (CVC, roll shifting, paircrossing, etc.). Os modelos de cálculo de carga aplicados aqui são baseados no método dos Blocos, em que se baseiam o modelo Bland-Ford com correção de arco de Hitchcock e o modelo Noncirc (desenvolvido por um dos autores ${ }^{3}$ ) que considera a deformação real do arco de contato para tiras finas e resistentes.

\section{MODELOS MATEMÁTICOS E NUMÉRICOS}

Quando se trata da determinação da coroa emergente de tiras laminadas, o conhecimento da correta deformação dos cilindros de laminação torna-se crucial, quanto maior a resistência do material da tira e a menor sua espessura. São apresentados brevemente, a seguir, dois modelos computacionais que determinam a deformação dos cilindros e os dois modelos de cálculo de carga utilizados.

\subsection{Método dos Coeficientes de Influência (MCl)}

Para se determinar o perfil da tira emergente, é necessário calcular o perfil deformado do cilindro de trabalho, que por sua vez depende do carregamento imposto aos cilindros. Assume-se, por hipótese, que a forma dada à tira emergente é idêntica à forma deformada do cilindro de trabalho. Shohet e Townsend ${ }^{4}$ desenvolveram um método que calcula a deflexão elástica dos cilindros devido à flexão e ao cisalhamento através dos coeficientes de influência, discretizando a chapa e os cilindros em fatias de larguras iguais.

Uma vez determinados tais coeficientes, montam-se as equações de cálculo da deflexão para cada cilindro, superpondo os deslocamentos por flexão, cisalhamento e achatamento localizado. Por meio das equações de equilíbrio de cada cilindro e de compatibilidade nas interfaces cilindro/cilindro e cilindro/tira, determinam-se as cargas distribuídas e o perfil deformado da tira, iterativamente.

Resumidamente o $\mathrm{MCl} /$ Berger funciona da seguinte maneira: a deformação dos cilindros é determinada baseado nas equações básicas da resistência dos materiais para vigas esbeltas e na teoria da elasticidade para determinação do achatamento localizado nos contatos entre tira/cilindro de trabalho e cilindro de trabalho/cilindro de encosto. Assim, inicialmente impõe-se uma carga uniforme sobre o modelo 4-high e calcula-se a deformação. Esta, por sua vez, é informada ao modelo de cálculo de carga (Bland-Ford ${ }^{5}$ com correção de Hitchcock ${ }^{6}$ ou um modelo mais sofisticado). Calcula-se a nova carga de laminação e se reaplica ao modelo 4-high de deformação dos cilindros, resultando em um novo perfil. Este ciclo iterativo é repetido até que a variação do perfil da tira entre a iteração atual e a anterior seja menor que uma tolerância. O programa MCl/Berger permite aplicar cargas de rollbending, e coroas mecânicas, de desgaste e térmicas.

O modelo desenvolvido por Pawelski e outros ${ }^{7}$ aplica o modelo de achatamento de cilindros desenvolvido por Berger ${ }^{8}$, possibilitando, assim, obter com boa precisão 0 perfil da queda brusca da tira junto às bordas (ombro ou edge-drop).

O modelo apresentado por Pawelski e seus colegas foi implementado com algumas modificações, e programado em linguagem do MATLAB. O programa denominado $\mathrm{MCl} /$ Berger, em reconhecimento a Bernd Berger, autor do modelo de achatamento de cilindros em contato utilizado no programa, foi testado e validado. 


\subsection{Modelo tridimensional em elementos finitos e cálculo de carga baseado em um modelo de blocos (MEF 3D/Blocos)}

O modelo $\mathrm{MCl} /$ Berger mostra-se bastante rápido para calcular a coroa emergente da tira, porém possui algumas limitações que devem ser consideradas, como, por exemplo, o fato dos cilindros não serem esbeltos como se assume neste simulador. Outra limitação deste modelo é o fato de não permitir a simulação de laminadores com cilindros com deslocamento axial e nem com perfis CVC.

Assim, propôs-se um modelo mais próximo à realidade dos laminadores mais sofisticados via modelagem em elementos finitos tridimensionais, porém mantendose o acoplamento com um modelo de cálculo da carga de laminação.

Este modelo, chamado aqui de MEF 3D/Blocos foi desenvolvido e validado com resultados experimentais.

Resumidamente, o modelo funciona exatamente como explicado no item anterior para o MCl/Berger, substituindo-se o cálculo da deformação dos cilindros por um modelo superior baseado em elementos finitos.

Devido às simetrias do laminador, somente a metade da metade superior do equipamento é modelada em elementos finitos, conforme a Figura 1.

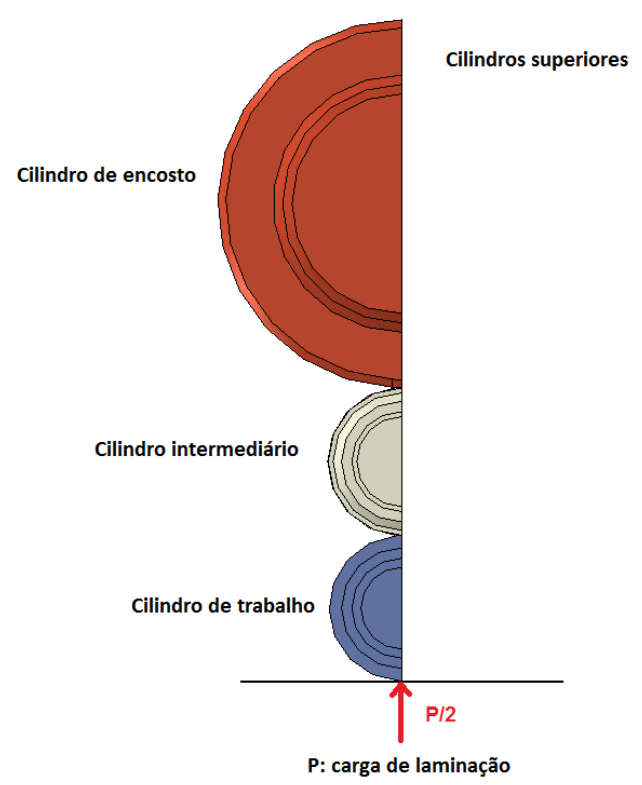

Figura 1. Modelo em elementos finitos.

\subsection{Modelos de cálculo da carga de laminação}

Von Karman ${ }^{9}$ desenvolveu o conceito de compressão vertical homogênea de segmentos da tira laminada em estado plano de deformação, reduzindo-o a um modelo 2D e em um problema 1D matemático. Além disso assumiu que o contato entre o cilindro de trabalho e a tira se manteriam circulares, levando a uma solução mais simples com menos erros para casos mais comuns. O coeficiente de atrito foi mantido constante. Assim foi possível obter uma solução analítica simples. A transformação da colina de atrito em um perfil semi-elíptico de mesma resultante permitiu conservar a forma circular do arco de contato (segundo Hitchcock) com um raio maior. Uma das soluções mais utilizadas na laminação é a dada por Bland e Ford com correção do raio do cilindro de trabalho via Hitchcock. Considerando que o aumento do valor da tensão de escoamento à medida em que a tira avança para 
dentro do laminador é pequena quando comparada à variação da pressão, foi possível obter uma solução simples e incluindo as tensões à ré e à frente.

Um fator crítico, porém, nesse modelo, é a hipótese da manutenção do arco circular, que em situações de laminação de tiras de maior resistência e espessuras finas, pode levar a resultados bastante imprecisos. Wiklund e Sandberg ${ }^{10}$ apresentam 0 chamado "fator de risco de achatamento" - flattening risk factor $\alpha=\mathrm{L} / \mathrm{hi}$ (onde L é 0 comprimento do arco de contato e hi a espessura da tira à entrada) Quando $\alpha>10$ espera-se um achatamento elevado do cilindro de trabalho, situação em que a utilização da aproximação de Hitchcock poderia ser questionada.

Nas situações em que o modelo de Bland-Ford-Hitchcock não pode ser utilizado com precisão, deve-se recorrer a modelos mais sofisticados em que se utiliza o arco real deformado na solução da equação de von Karman.

Para as situações em que a solução de Bland-Ford não for adequada deve-se recorrer a modelos que levem em consideração a deformação real do cilindro, que não é circular. Para essas situações foi desenvolvido o modelo Noncirc. Trata-se de um modelo baseado no método dos blocos aplicado a cada uma das cinco regiões que podem ocorrer dentro de um arco de contato na laminação (1-região elástica de entrada, 2- região plástica pós-elástica, 3- região de deformação plástica contida, 4região plástica, e 5- região elástica de saída), e que utiliza os valores reais de deformação do cilindro dentro do contato tira-cilindro, conforme a Figura 2.

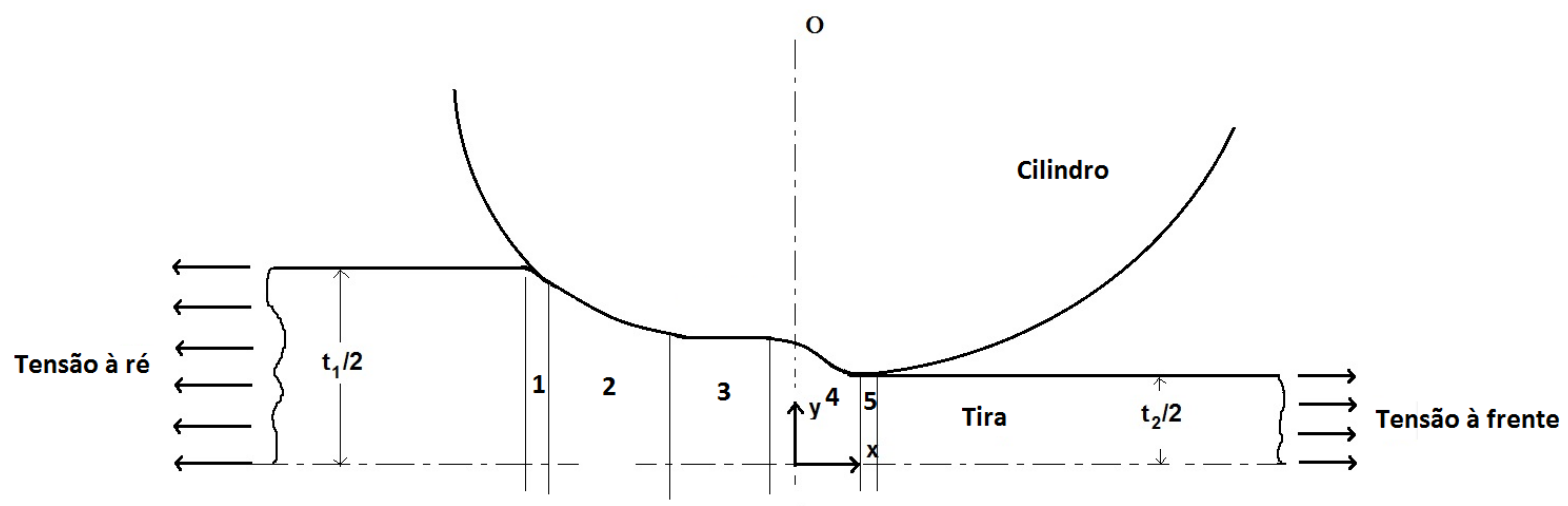

Figura 2. Regiões dentro do arco de contato (modelo Noncirc).

\section{EXPERIMENTOS}

Para ajustar os modelos citados anteriormente, foram realizados experimentos no Laminador de Tiras a Frio no. 1 (LTF1) e no Laminador de Encruamento (LE) da CSN.

O LTF1 é constituído de 5 cadeiras, sendo a primeira um 6-high e as 4 restantes, 4high. O LE é constituído de duas cadeiras 4-high.

Em cada experimento foi realizada uma parada rápida durante uma campanha, e então foram retiradas amostras da tira à entrada e à saída e entre as cadeiras. Por meio de um equipamento medidor de espessuras por ultrassom, foram realizadas medições da distribuição das espessuras ao longo da largura de cada amostra, e também foram realizados ensaios de tração para determinar a curva de escoamento do material.

Para cada bobina foram recolhidas todas as informações operacionais, tais como carga de laminação, tensões à ré e à frente, velocidade do cilindro, valores de carga de roll bending, diâmetros dos cilindros e suas coroas, etc. 
Tendo em mãos também a geometria detalhada dos laminadores, foi possível ajustar os modelos $\mathrm{MCl} /$ Berger e MEF 3D/Blocos. A partir da carga de laminação medida, foi determinado o coeficiente de atrito médio.

\section{RESULTADOS E DISCUSSÃO}

São apresentados, a seguir, alguns dos resultados preliminares deste trabalho. Pode-se observar na Figura 3 o modelo em elementos finitos da primeira cadeira do LTF1, um 6-high com cilindros intermediários deslocáveis axialmente. Foi utilizado o programa de elementos finitos Simulia Abaqus.

As condições operacionais estão apresentadas na Tabela 1.

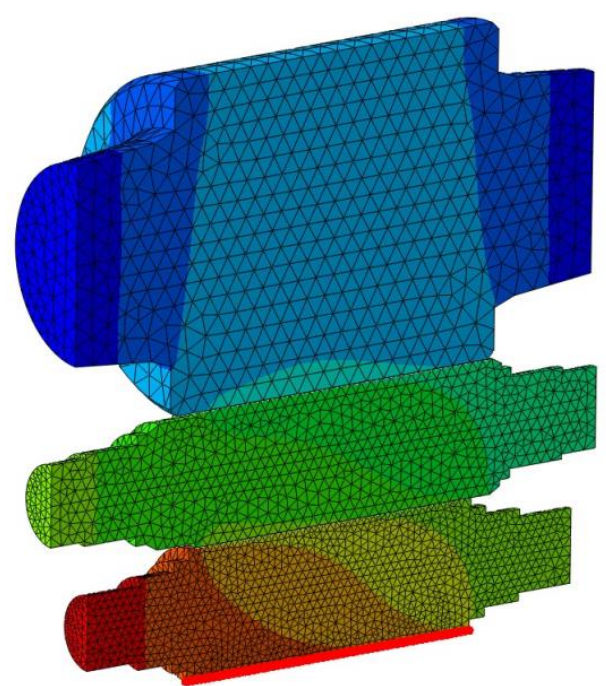

Figura 3. Modelo em elementos finitos.

Tabela 1. Dados operacionais do experimento no LTF1

\begin{tabular}{cc}
\hline Parâmetro & Valor \\
\hline Espessura de entrada da tira $(\mathrm{mm})$ & 2.20 \\
Espessura de saída da tira $(\mathrm{mm})$ & 1.34 \\
Largura da tira $(\mathrm{mm})$ & 980 \\
Raio do cilindro de trabalho $(\mathrm{mm})$ & 200 \\
Raio do cilindro de encosto $(\mathrm{mm})$ & 600 \\
Raio do cilindro intermediário $(\mathrm{mm})$ & 210 \\
Curva de escoamento $(\mathrm{MPa})$ & $\mathrm{Y}=150+350 \varepsilon^{0.5}$ \\
Tensão de tração à frente $(\mathrm{MPa})$ & 25 \\
Tensão de tração à ré $(\mathrm{MPa})$ & 5 \\
Coeficiente de atrito & 0.4 \\
Deslocamento axial $(\mathrm{mm})$ & 70 \\
\hline
\end{tabular}

O resultado do perfil da tira devido à simulação foi comparado com o medido, e está representado na Figura 4. 


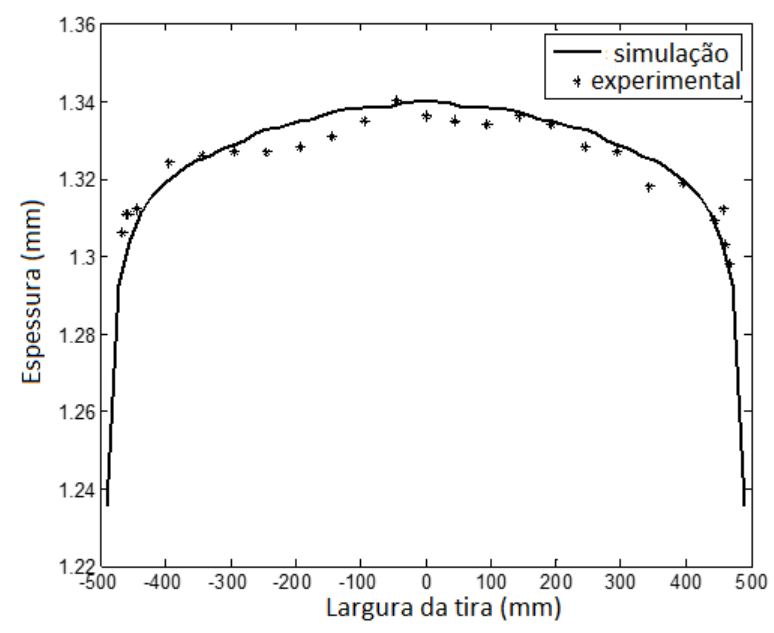

Figura 4. Comparativo dos resultados do perfil.

Uma das cadeiras do LE foi modelado no programa MCl/Berger, e houve problemas na convergência do mesmo para as condições operacionais impostas. Isso se deveu, em parte, ao fato do modelo de carga (Noncirc) aplicado não estar preparado para simular esse tipo de laminação particular, em que a redução é extremamente pequena, o que causa uma elevada carga na primeira iteração em um arco extremamente pequeno. Isso, porém, não impediu de realizar algumas simulações com condições parecidas e, qualitativamente, obter o comportamento do perfil da tira diante de diferentes coroas mecânicas nos cilindros e sob variadas cargas de roll bending. Dessa forma foi possível aplicar uma coroa mecânica sobre o cilindro de encosto de uma das cadeiras do LE e verificar experimentalmente uma redução na produção de defeitos de ondulado lateral. Pode-se observar na Figura 5 os perfis emergentes da tira em função das cargas de roll bending.

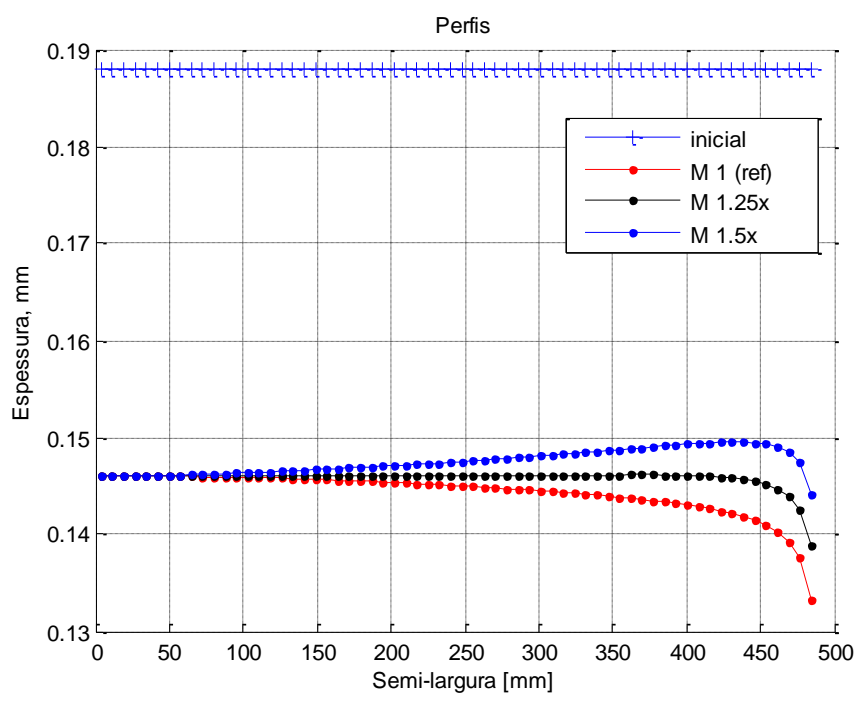

Figura 5. Perfis emergentes em função dos valores de roll bending.

\section{CONCLUSÃO}

O presente trabalho apresenta alguns dos resultados preliminares da aplicação de dois modelos matemáticos de simulação do processo de laminação a frio de tiras na CSN. Ambos os modelos calculam o perfil emergente da tira considerando a 
deformação elástica dos cilindros, um baseado em um modelo de coeficientes de influência e outro um modelo em elementos finitos.

O primeiro resultado apresentado para a primeira cadeira do LTF1, um 6-high, mostrou boa aproximação com o resultado experimental. O modelo em CAD respeita fielmente os desenhos dos cilindros e seu posicionamento. O modelo rodou todas as iterações em 20 minutos em um computador desktop com doze núcleos, processador Intel Xeon. $O$ reduzido tempo de processamento mostra também que 0 modelo é promissor, em especial para casos mais exigentes, como é o caso de tiras finas e de material de elevada resistência.

O segundo resultado, apesar de ainda ser inconclusivo, levou à aplicação de uma coroa nos cilindros do LE e resultou na redução dos defeitos de ondulado lateral.

Ainda há muito a se fazer para se chegar a resultados melhores, mas os presentes resultados demonstram não só a viabilidade disso como também seu valor.

\section{Agradecimentos}

Os autores agradecem ao Conselho Nacional de Pesquisa e Desenvolvimento Científico e Tecnológico, CNPq, processo 488027/2013-6, sem o qual não seria possível realizar o presente trabalho. E também à FAPEMIG, ao CEFET-MG e à CSN, Instituição parceira deste projeto, pelo apoio à sua realização.

\section{REFERÊNCIAS}

1 Y. Shigaki, Um estudo da laminação de tiras e chapas planas através de um modelo matemático-numérico de um laminador quádruo, Tese de Doutorado, Programa de Pós-Graduação em Engenharia Metalúrgica e de Minas da UFMG, 2001.

2 K. Linghu, Z. Jiang, J. Zhao, F. Li, D. Wei, 3D FEM analysis of strip shape during multipass rolling in a 6-high CVC cold rolling mill, Int. J. Adv. Manuf. Tech. 2014, On-line First 1-13.

3 Shigaki Y., Nakhoul R., Montmitonnet P., Numerical treatments of slipping/no-slip zones in cold rolling of thin sheets with heavy roll deformation, Lubricants, 2015, 3, 113-131.

4 Shohet K. N. e Townsend N. A., Roll bending methods of crown control in four-high plate mills, J. Iron and Steel Inst., novembro 1968, 1088-1098.

5 Bland D.R., Ford H., The calculation of roll force and torque in cold strip rolling with tensions, Proc. Inst. Mech. Eng., 1948, 159, 144-153.

6 Hitchcock J.H., Roll neck bearings. Report, ASME Research Committee, 1935.

7 Pawelski O., Rasp W., Teutsch H., A mathematical model with a combined analytical and numerical approach for predicting the thickness profile of a cold-rolled strip, Steel Res., 1985, 56, 6, 327-331.

8 Berger, B.; Pawelski, O.; Funke, P. Die beeinflussung des dickenprofils von bändern und Blechen durch walzenbiegeeinrichtungen, 1976, Stahl und Eisen, 96, No. 8, pp. 377-381.

9 von Karman T., Beitrag zur Theorie des Walzvorganges, Z. Angew. Math. Mech., 1925, 5, 139-141.

10 Wiklund O., Sandberg F., and Lenard J. G., Modelling and control of temper rolling and skin pass rolling. 2002, Metal Forming Science and Practice (Elsevier Science Ltd), Oxford, 313-343 